\title{
Erros redacionais em prescrições médicas em uma policlínica de Manaus, Amazonas, Brasil
}

\author{
Physicians presciptions mistakes in a polyclinic \\ of Manaus, Amazonas, Brazil
}

Jackeline de Souza MARINHO; Janayna Freitas de Araújo LIBÓRIO; Herbert Theury Souza COSTA; Ana Cláudia Silva

SANTOS; Fernanda GUILHON-SIMPLICIO

Faculdade de Ciências Farmacêtticas, Universidade Federal do Amazonas, Rua Alexandre Amorim, 330, Aparecida, CEP 69010-300, Manaus, Amazonas, Brasil.

E-mail:fgsfarmaceutica@gmail.com

\begin{abstract}
Writing errors in physicians prescriptions are an unfortunate reality in many health facilities, hampering the dispensing of medicaments by pharmacists and consequently harming the user pharmacotherapy. The overall aim of this study was to identify and quantify the most common writing errors found prescriptions received in a Public Polyclinic located in central Manaus, capital of Amazonas State. The evaluation was carried out from January 2012 to April 2012, and the results were compared with those obtained by other similar studies. The main errors found (occurrence above $10.00 \%)$ in the 378 prescriptions analyzed were illegible user name $(14.40 \%)$, the illegible drug concentration $(14.40 \%)$, lack of drug concentration (14.16\%), illegible drug name (12.58\%) and unreadable dosage (10.58\%). All highlighted writing errors may be directly implicated in the incorrect dispensing of medicines and consequent treatment failure. These results show that, despite the publication of several laws governing the writing of prescriptions, additional education measures are still necessary, involving the entire interdisciplinary team of health professionals in order to minimize the occurrence and consequences of these errors and ensure efficient pharmacotherapy.
\end{abstract}

Key Words: drug prescriptions, drug legislation, pharmaceutical care

\section{RESUMO}

Os erros redacionais em prescrições médicas são uma realidade lamentável em muitas unidades de saúde brasileiras, que dificultam a dispensação correta e, consequentemente, podem prejudicar a farmacoterapia do usuário. O objetivo do presente estudo foi identificar e quantificar os erros de redação encontrados nas prescrições médicas recebidas em uma Policlínica Estadual situada na zona central de Manaus, capital do Estado do Amazonas, no período de janeiro a abril de 2012. Os principais erros redacionais encontrados (ocorrência acima de 10,00\%) nas 378 prescrições analisadas foram: ilegibilidade do nome do usuário $(14,40 \%)$, ilegibilidade da concentração do fármaco $(14,40 \%)$, ausência da concentração do fármaco (14,16\%), ilegibilidade do nome do medicamento $(12,58 \%)$ e ilegibilidade da posologia (10,58\%). Tais resultados mostram que, apesar da publicação de diversas legislações que regulamentam a redação de prescrições médicas, ainda são necessárias medidas educativas e ações interdisciplinares envolvendo toda a equipe de profissionais da saúde para minimizar a ocorrência e as consequências desses erros, garantindo uma farmacoterapia eficiente.

Palavras-Chave: prescrições de medicamentos, legislação de medicamentos, atenção farmacêutica 


\section{INTRODUÇÃO}

As prescrições manuscritas têm sido o principal meio de informar ao farmacêutico da terapêutica recomendada pelo médico ao paciente. Contudo, esse elo de comunicação está sujeito a riscos como ilegibilidade, má interpretação e falsificações (1). Nesse contexto, tem sido uma tendência mundial a adoção de sistemas de prescrição eletrônica. Tais sistemas, onde a prescrição é transmitida por meio de um computador ou dispositivo móvel do consultório médico à farmácia ou drogaria (2), tem como proposta primordial a redução de erros de medicação causados no momento da prescrição, aumentando a eficiência do processo e a segurança do paciente (3). Entretanto, essa não é uma realidade abrangente no sistema de saúde do Brasil, cuja maioria das unidades de saúde ainda utiliza prescrições médicas manuscritas.

A prescrição envolve aspectos legais, técnicos e clínicos. Ao prescritor cabe a responsabilidade da transmissão das informações pertinentes, com apropriada clareza, a todos os profissionais que utilizem esse documento, o que tem importante impacto na promoção do uso racional de medicamentos (4). Quando se analisa a legislação brasileira, considera-se parte essencial de uma prescrição: nome do usuário, data, nome do medicamento a ser administrado, concentração, dosagem, via de administração, frequência, uso de pesos e medidas oficiais e a assinatura e registro no conselho regional de medicina $(5,6)$. Em muitas farmácias e drogarias é adotada como protocolo a recomendação ao usuário que volte ao médico e solicite uma nova receita ou a leitura da mesma, em caso de ausência ou ilegibilidade de algum desses itens, ato que, apesar de amparado pela Agência Nacional de Vigilância Sanitária (6), em geral causa mal estar ao usuário e aos profissionais envolvidos.

A mesma resolução recomenda que os medicamentos não sejam dispensados em caso de receitas ilegíveis ou que induzam a erro ou confusão. Na maioria das vezes, porém, o responsável pelo atendimento dispensa o medicamento conforme sua interpretação, o que coloca em risco a saúde e a vida do usuário e aumenta o custo do tratamento, em caso de troca de medicação (7). Portanto, esse é um tema de clara importância socioeconômica, o que é enfatizado pelas inúmeras discussões suscitadas em todo o país acerca do tema, que muitas vezes leva à redação de novas legislações.

O Conselho Federal de Medicina do Brasil publicou a resolução ${ }^{\circ} 1931$ em 2009, que proíbe o médico de receitar, atestar ou emitir laudos de forma secreta ou ilegível (8). Além disso, em alguns Estados brasileiros já foram decretadas leis que exigem que todas as prescrições médicas e odontológicas sejam digitadas em computador ou escritas manualmente em letra de imprensa, forma ou caixa alta, como no caso do Estado de São Paulo (Lei n $\left.{ }^{\circ} 10.241 / 1999\right)$ (9), Paraná (Lei no 13.556/2002) (10), Amazonas (Lei n⿳3199/2007) (11) e Paraíba (Lei no 9373/2011) (12).

Apesar disso, é notório o descompasso entre a teoria e a realidade em diversas Instituições de saúde brasileiras. Diante do exposto, este trabalho realizou um levantamento dos principais erros redacionais encontrados em prescrições médicas atendidas em uma Policlínica Estadual sediada na zona central de Manaus, comparando os resultados encontrados com os de estudos semelhantes realizados em outras unidades de saúde do país, para elaborar um inédito panorama que pode corroborar para a formulação e aplicação de medidas preventivas / educativas mais adequadas.

\section{MÉTODO}

\section{Tipo de estudo}

Trata-se de um estudo descritivo transversal que avaliou prescrições médicas recebidas no serviço de dispensação de uma Policlínica Estadual da cidade de Manaus, Amazonas, quanto à obediência a critérios redacionais preconizados pelas legislações vigentes.

\section{Local de estudo}

O estudo foi desenvolvido na farmácia da Policlínica Estadual Governador Gilberto Mestrinho, localizada na zona central da cidade de Manaus, capital do Estado do Amazonas, que acolhe, além de prescrições médicas da própria unidade, as prescrições de unidades de saúde do todo o Estado do Amazonas, independentemente de serem públicas ou privadas.

\section{Amostragem e Casuística}

Foi realizado um levantamento dos principais erros de redação encontrados nas segundas vias de prescrições médicas (cópias carbonadas ou fotocópias da original) retidas na farmácia da Policlínica Governador Gilberto Mestrinho, no período de janeiro a abril de 2012.

Da população de 22.564 prescrições, foi analisado um total de 378 prescrições manuscritas e digitadas, que correspondem à amostra significante, com nível de confiança de $95 \%$, calculada por meio da fórmula abaixo (13):

$$
n=\text { N. } Z^{2} \text {. P. }(1-p) /\left[Z^{2} \text {. P. }(1-p)+\mathrm{e}^{2} .(\mathrm{N}-1)\right]
$$

onde:

$n=$ amostra calculada

$\mathrm{N}=$ população (22.564 prescrições que foram recebidas nos meses que serão realizados o estudo)

$\mathrm{Z}=$ variável normal padronizada associada ao nível de segurança (para intervalo de confiança de 95\%, correspondente a 1,96 na tabela de distribuição normal) 
$\mathrm{p}=$ verdadeira probabilidade do evento (por ser desconhecida, adotou-se $50 \%$, que é a probabilidade de qualquer evento ocorrer)

$\mathrm{e}=$ erro amostral (adotou-se 5\%, valor comum nesse tipo de estudo, que em geral é definido pelo próprio pesquisador) (13).

As prescrições foram selecionadas de modo aleatório simples, por meio de sorteio, sem comprometimento dos resultados e sem imposição hierárquica entre os meses e entre os tipos de prescrições analisadas (manuscritas ou digitadas), adotando-se os seguintes critérios:

Critérios de exclusão: as prescrições de estudo foram exclusivamente médicas. As prescrições que foram analisadas não englobaram os receituários de medicamentos de controle especial enquadrados nas RDC 344/98 (14) da Anvisa, que possuem uma dinâmica diferenciada sob a legislação brasileira. Foram excluídas prescrições as quais a segunda via encontrava-se de difícil visualização.

Critérios de inclusão: prescrições contendo medicamentos das demais classes terapêuticas, podendo ser manuscritas (totalmente escritas à mão) ou digitadas (redigidas com uso de computador, tendo manuscrita somente a assinatura do prescritor) direcionadas a usuários de todas as faixas etárias.

\section{Análise das variáveis estudadas}

As 13 variáveis que foram analisadas neste estudo foram determinadas com base em itens mínimos de uma prescrição médica preconizados pelas legislações brasileiras existentes $(5,6)$, a saber:

a) Abreviações do nome do medicamento (considerando a Denominação Comum Brasileira - DCB) (15): quando o nome do medicamento apresentava-se como abreviaturas ou siglas, por exemplo, "HZT" ao invés de hidroclorotiazida.

b) Ausência da assinatura do médico: prescrição que não apresentava a assinatura do médico.

c) Ausência da concentração do medicamento: prescrição cuja concentração do medicamento estava ausente.

d) Ausência da data da prescrição: prescrição que não apresenta registro de data.

e) Ausência da posologia: prescrição que não apresentava qualquer tipo de informação posológica.

f) Ausência do número do registro profissional: prescrição que não apresentava o número do registro profissional do médico.

g) Concentração incompleta do medicamento: prescrição que apresentava a concentração sem as unidades de medida. h) Grafia incorreta do nome do medicamento: quando a prescrição apresentava o nome do medicamento com a grafia incorreta, por exemplo, "amoxilina" no lugar de amoxicilina.

i) Ilegibilidade da concentração do medicamento: quando a concentração apresentava-se ilegível ou permitia dúvidas quanto aos números e/ou unidades de medidas apresentados.

j) Ilegibilidade da posologia: quando a posologia apresentava-se ilegível ou de difícil entendimento.

k) Ilegibilidade do nome do medicamento (considerando a Denominação Comum Brasileira - DCB) (15): quando a escrita do nome do medicamento apresentava-se ilegível ou de difícil entendimento.

l) Ilegibilidade do nome do usuário: quando o nome do usuário apresentava-se ilegível ou de difícil entendimento.

m) Rasuras: quando a prescrição apresentava qualquer rasura em toda a sua extensão que dificultasse seu entendimento.

A ilegibilidade foi analisada somente nas prescrições manuscritas, e considerada quando dois observadores não conseguiram interpretar a grafia médica. Além desses fatores, também foi analisado o número de prescrições médicas que apresentaram pelo menos um erro, dentre as manuscritas e digitadas.

Os erros redacionais encontrados foram transcritos manualmente para o formulário de coleta de dados, e posteriormente digitalizados para a realização das análises estatísticas. As prescrições foram identificadas pelo número correspondente à sequência em que foram sorteadas, e não foram compilados os nomes dos pacientes ou prescritores para o instrumento de coleta, apenas os erros de grafia contabilizados nesses itens, garantindo, portanto, o anonimato dos sujeitos envolvidos na pesquisa. Em suma, em nenhum momento do estudo foi analisado ou divulgado o nome dos usuários ou prescritores, sendo esses dados utilizados somente para a verificação das variáveis $\mathbf{b}, \mathbf{f}$ e $\mathbf{l}$.

\section{Análise dos resultados}

Os dados foram transcritos para meio eletrônico e submetidos a análises estatísticas descritivas e os resultados apresentados como frequências simples e percentuais. Os dados obtidos foram comparados aos resultados de estudos semelhantes realizados em outras unidades de saúde brasileira.

\section{Aspectos éticos}

O presente estudo foi submetido à avaliação do Comitê de Ética em Pesquisa com Seres Humanos da Universidade Federal do Amazonas (CEP/UFAM) com 
solicitação de dispensa do Termo de Compromisso Livre e Esclarecido (TCLE) e obteve parecer favorável em 01 de março de 2013 (CAAE n ${ }^{\circ}$ 12310913.0.0000.5020).

\section{RESULTADOS E DISCUSSÃO}

Das 378 prescrições médicas analisadas, 307 $(81,2 \%)$ eram manuscritas e $71(18,8 \%)$ eram digitadas. Do total, 266 prescrições $(70,3 \%)$ apresentaram pelo menos um dos erros redacionais analisados, portanto, não atendiam aos pré-requisitos técnicos e legais para uma dispensação eficiente e utilização correta dos medicamentos, estando em desacordo com os preceitos do
Uso Racional de Medicamentos. Como esperado, a porcentagem de erros nas prescrições manuscritas $(79,8 \%)$ foi muito maior que nas digitadas $(29,7 \%)$, visto que nas últimas não foram aplicados critérios de ilegibilidade.

O número do total de erros detectados foi de 699 , o que representa uma média de 1,8 erros por prescrição médica, sendo os mais quantitativamente relevantes (com ocorrência acima de 10,00\%), ilegibilidade da concentração do medicamento $(14,40 \%)$, ilegibilidade do nome do usuário $(14,40 \%)$, ausência da concentração do medicamento $(14,16 \%)$, ilegibilidade do nome do medicamento $(12,58 \%)$ e ilegibilidade da posologia $(10,58 \%)$, como pode ser observado na Tabela 1.

Tabela 1. Ocorrência de erros redacionais nas prescrições atendidas na Farmácia Ambulatorial da Policlínica Gilberto Mestrinho (Manaus, Amazonas) no período de janeiro abril de 2012.

\begin{tabular}{|l|c|c|}
\hline \multicolumn{1}{|c|}{ ERROS REDACIONAIS CONSIDERADOS } & FREQUÊNCIA & FREQUÊNCIA RELATIVA (\%) \\
\hline Abreviações do nome do medicamento & 15 & 2,14 \\
\hline Ausência da assinatura do médico & 9 & 1,28 \\
\hline Ausência da concentração do medicamento & 99 & 14,16 \\
\hline Ausência da data da prescrição & 67 & 9,58 \\
\hline Ausência da posologia & 5 & 0,71 \\
\hline Ausência do número de registro profissional & 15 & 2,14 \\
\hline Concentração incompleta do medicamento & 59 & 8,44 \\
\hline Grafia incorreta do nome do medicamento & 48 & 6,86 \\
\hline Ilegibilidade da concentração do medicamento & 101 & 14,44 \\
\hline Ilegibilidade da posologia & 74 & 10,58 \\
\hline Ilegibilidade do nome do medicamento & 88 & 12,58 \\
\hline Ilegibilidade do nome do usuário & 101 & 14,44 \\
\hline Rasuras & 18 & 2,57 \\
\hline Total & 699 & 100,00 \\
\hline
\end{tabular}

Em consulta a algumas bases de dados de revistas científicas nacionais não foram encontrados estudos semelhantes a este realizados em outras unidades de saúde públicas da Região Norte do Brasil, o que por um lado dificulta a discussão dos resultados, mas evidencia a importância da realização de pesquisas como esta. Por isso, apesar das discrepâncias socioeconômicas entre as regiões brasileiras, os resultados desta pesquisa foram comparados aos resultados encontrados em unidades de saúde de outros Estados, visando traçar um panorama da realidade local, ainda que preliminar, que pode servir de parâmetro para futuros estudos e para elaboração de estratégias para eliminar os principais erros redacionais encontrados.

Um estudo realizado por Lins et al. (2012) em uma unidade de saúde de um município de Santa Catarina constatou que 363 prescrições $(82,00 \%$ das prescrições analisadas no estudo) continham um ou mais erros redacionais (16). Em outro estudo realizado em uma unidade básica de saúde de Aracaju-SE foi constatado que $71,50 \%$ das prescrições avaliadas não atendiam às exigências (17). Estes resultados são relativamente próximos ao encontrado neste estudo $(70,30 \%)$, tornando evidente que esse é um problema que permeia diferentes regiões do país.

Quanto ao uso de abreviações e siglas, o resultado encontrado neste estudo $(2,14 \%)$ foi inferior ao encontrado por Dantas (2008) em uma unidade de saúde de Fortaleza-CE (18), Guzzato et al.(2007), em uma unidade de Porto Alegre-RS (19) e Frick et al. (2010) em outra unidade à noroeste do Estado do Rio Grande do Sul (20), que revelaram um percentual de 7,00\%, 6,30\% e 7,96\%, 
respectivamente. $\mathrm{O}$ uso de siglas pode refletir uma tentativa dos prescritores em acelerar o atendimento, aspecto cada vez mais exigido no sistema de saúde público e privado, devido à alta demanda e baixa remuneração dos profissionais médicos e a crescente priorização da redução de custos, em detrimento ao bem estar do usuário (21). Contudo, por não serem padronizadas ou serem de conhecimento restrito ao ambiente acadêmico, o uso dessas abreviaturas e siglas pode dificultar ou inviabilizar o atendimento da prescrição (22).

Por outro lado, não foram encontrados estudos que levassem em consideração erros referentes à grafia incorreta do nome do medicamento, que neste trabalho apresentaram uma ocorrência significativa $(6,86 \%)$. A escolha dessa variável para o estudo levou em consideração a realidade local, onde muitas vezes a dispensação de medicamentos não é feita por farmacêuticos, mas por pessoas com os mais diversos níveis de escolaridade, com pouco ou nenhum conhecimento técnico sobre os medicamentos. Entende-se que nessas condições, a prescrição médica pode não ser atendida equivocadamente, o que de fato é evitado pela intervenção do farmacêutico, que por sua vez, pode detectar o problema e orientar o paciente de maneira mais eficiente.

Erros envolvendo ausência da assinatura do prescritor $(1,28 \%)$ e ausência do número do registro profissional $(2,14 \%)$ também foram inferiores aos estudos realizados por Sebastião (2002) em Ouro Preto-MG (23) e Néri (2004) em Fortaleza-CE (24), ambos realizados em farmácias hospitalares. Contudo, apesar desses índices relativamente baixos, a ausência de tais dados não pode ser negligenciada, uma vez que pode levar a duplicidade e falsificação de prescrições, bem como desvios de medicamentos (17). Além disso, dificulta a desejável interação entre os profissionais de saúde, que é sempre benéfica ao usuário (25).

De forma semelhante, a ilegibilidade do nome do usuário $(14,40 \%)$ pode facilitar desvios de medicação. No estudo de Rosa et al. (2008) em uma farmácia hospitalar no Estado de Minas Gerais foi detectado que em $47,00 \%$ das prescrições analisadas, o nome do usuário estava incompleto e/ou ilegível (26). No Rio Grande do Sul, Frick et al. (2010) encontraram um índice de $15,93 \%$ quando analisou o mesmo parâmetro (20). Essa diferença pode ser justificada pelo fato que, no presente estudo, não ter sido analisado se o nome do paciente estava completo, por se tratar de um estudo retrospectivo, no qual não houve contato com o documento de identificação do paciente. Contudo, no estudo que Araújo \& Uchôa (2011) realizaram em farmácia hospitalar no Rio Grande do Norte, considerando apenas a ilegibilidade do nome do paciente, foi constatado um porcentual de $21,95 \%$ (27), mais próximo do encontrado neste estudo.
Dantas (2008) em Icapuí-CE, e Guzzato et al. (2007) em Porto Alegre-RS, identificaram, respectivamente, que $31,00 \%$ (18) e $36,70 \%$ (19) das prescrições que analisaram não continham a concentração do medicamento, resultado bem superior ao deste estudo $(14,16 \%)$. Por outro lado, o estudo realizado por Lins et al. (2012), em uma unidade básica de saúde de um município do meio-oeste de Santa Catarina, apontou uma porcentagem de $17,60 \%$ (16), bastante semelhante à encontrada nesta pesquisa. Quando uma prescrição não apresenta a concentração do medicamento não se deve realizar a dispensação, visto que para um mesmo medicamento podem existir diversas concentrações (28).

Da mesma forma, problemas como concentração incompleta $(8,40 \%)$ e ilegibilidade da mesma $(14,40 \%)$ apresentaram porcentagens bastante relevantes, sendo o primeiro dado um pouco inferior ao resultado encontrado por Guzzato et al. (2007) em Porto Alegre-RS que foi de $11,70 \%$ (19). Sabe-se que a ausência da unidade de medida na concentração, bem como a ilegibilidade da mesma, podem comprometer a dispensação do medicamento ao paciente, ou até mesmo induzir o paciente a erro na administração, o que pode resultar em uma intoxicação medicamentosa. A ilegibilidade do próprio nome do medicamento também mostrou uma porcentagem bastante representativa (12,50\%) neste estudo. Lyra Junior et al. (2004), que utilizaram uma amostra de prescrições maior em um estudo realizado em Aracaju-SE, obtiveram o porcentual de 24,00\% (17). Este aspecto é bastante relevante, visto que é crítico fator desencadeador de erros de dispensação.

Elevados índices de ausência de posologia foram encontrados por Percly et al. (2006) em prescrições ambulatoriais atendidas numa farmácia hospitalar na cidade do Rio de Janeiro-RJ (29), por Néri (2004) (24), e Aguiar et al. (2006) (7) em prescrições de hospitais de Fortaleza-CE, e por Frick et al. (2010) em um município da região noroeste do Rio Grande do Sul (18), os quais foram, respectivamente, $40,09 \%, 26,30 \%, 11,80 \%$ e $11,50 \%$. Esses resultados foram bastante discrepantes do encontrado neste estudo $(0,71 \%)$, o que pode ser explicado pelos critérios utilizados pelos autores para definir a ausência de posologia, que nesta pesquisa foi a ausência total de qualquer tipo de informação posológica. Lins et al. (2012), utilizando o mesmo critério adotado neste estudo, encontrou a porcentagem de $0,80 \% \mathrm{em}$ uma unidade básica de Santa Catarina (16).

A posologia indica como e em que horários o paciente deve consumir o medicamento, assim, a ausência dessa informação pode levar o usuário a ingerir uma quantidade inferior ou superior ao necessário para alcançar as metas farmacoterapêuticas estabelecidas (30). 
O mesmo ocorre com a ilegibilidade da posologia $(10,58 \%)$, cujo índice encontrado neste estudo apresentou-se ligeiramente superior ao encontrado no estudo realizado por Dantas (2008) em Icapuí-CE, 7,00\% (18). A ilegibilidade da posologia pode gerar interpretações equivocadas, levando a troca de medicamentos, da via de administração, da dose prescrita, entre outros problemas (27).

A ausência de data nas prescrições $(9,50 \%)$ foi similar ao achado de Aguiar et al. (2006) que foi de 9,60\% (7), e superior ao de Néri (2004) que encontrou 3,50\% (24) das prescrições sem esta informação, ambos os resultados de estudos realizados em hospitais de Fortaleza-CE, e também superior ao de Frick et al. (2010), que encontraram 7,08\% em um estudo realizado no Rio Grande do Sul (20). Por outro lado, em uma unidade de saúde em Santa Catarina, Lins et al. (2010) encontraram um percentual de $0,80 \%(16)$, bastante inferior aos dados mencionados anteriormente.

Em pesquisa realizada em cinco hospitais universitários brasileiros pertencentes à Rede de Hospitais Sentinela da Anvisa, Gimenes et al. (2010), encontraram um valor próximo, de $0,90 \%$ (31). A data é um dado fundamental numa prescrição médica, pois auxilia na orientação do usuário, farmacêutico, equipe de enfermagem ou cuidador quanto ao período de utilização da medicação e validade da prescrição, dificultando o uso indiscriminado de medicamentos (7).

Outro critério bastante relevante é a presença de rasuras na prescrição médica. Esse estudo revelou um porcentual de $2,57 \%$ de rasuras nas prescrições analisadas, valor muito próximo ao encontrado por Dantas (2008) em prescrições ambulatoriais de um hospital em Fortaleza-CE, que foi de 3,00\% (18). Gimenes et al. (2010) que realizaram o estudo em quatro hospitais universitários, encontraram um porcentual superior, de $6,40 \%(31)$.

Muitas rasuras são feitas pelos próprios usuários, na tentativa de receber mais medicação. Está enraizada na sociedade atual que os medicamentos são a forma mais eficaz de promover a saúde, ao que se soma o sentimento de "direito adquirido", contexto no qual o medicamento é tratado como bem de consumo. Além disso, as longas filas e o atendimento cada vez mais desumanizado dos sistemas de saúde brasileiros desestimula o usuário a procurar a assistência de profissionais da saúde, o que tem impacto direto no desejo de acúmulo de medicamentos em residências e na automedicação (21).

No âmbito internacional, também é possível observar que erros em prescrições médicas são uma preocupação relevante. Na Índia, país com o mesmo grau de industrialização que o Brasil, 100,00\% de 290 prescrições de um Hospital Universitário analisadas em um es- tudo de 2013 apresentaram algum tipo de erro, incluindo ilegibilidade (32). Na Inglaterra, outro estudo de 2013 apontou que informações incompletas nas prescrições contabilizaram $30,00 \%$ dos erros encontrados em prescrições atendidas em três unidades básicas de saúde (33). Em um estudo realizado na Espanha com 42.000 prescrições, foi detectado que $26,20 \%$ eram ilegíveis, entre outros erros, especialmente omissão de dados (34).

Mesmo em países que adotam sistemas de prescrição eletrônica, ainda são significativos os índices de erros nesses documentos. Em um estudo que analisou prescrições eletrônicas atendidas em farmácias comunitárias dos Estados Unidos da América, 60,70\% das 3850 prescrições analisadas apresentaram alguma omissão de dados, principalmente dose e duração do tratamento. Abreviações inapropriadas de nome de medicamentos também apresentaram um índice bastante expressivo, de $52,00 \%(35)$. Na Suécia, país que utiliza prescrições eletrônicas em toda sua rede de atenção primária à saúde desde 1995, estudos apontam que $1 \%$ de todas as prescrições emitidas por ano ainda requerem que o farmacêutico esclareça informações obscuras junto ao prescritor (36).

No Brasil ainda são poucas as unidades de saúde que utilizam prescrições eletrônicas, a maior parte restrita a ambientes hospitalares. Em estudo realizado por Abramovicius (2007) com prescrições médicas do Hospital das Clínicas da Faculdade de Medicina de Ribeirão Preto-SP, o índice de erros foi de 9,05\%, incluídos os de prescrição e administrativos (37).

Destarte, o que se vem sendo discutido nos países onde a prescrição eletrônica é bem estabelecida, é que essa ferramenta acaba por infligir erros ou problemas diferentes dos associados às prescrições manuais, como dificuldades na interação homem-máquina que pode levar à troca de medicamentos, sobrecarga do tempo prescritor com o uso da tecnologia, perda de dados e demasiada dependência de tecnologia que, em determinadas circunstâncias, pode culminar no não atendimento ao paciente (38).

\section{CONCLUSÃO}

A prescrição médica eletrônica é frequentemente citada na literatura como uma das medidas mais eficazes para a redução dos erros de prescrição. Em fato, a utilização desse recurso poderia minimizar a presença de erros redacionais nas prescrições e eliminaria o problema da ilegibilidade. Contudo, é notório que esse é um sistema que demanda alto investimento, o que torna sua aplicação praticamente inviável em muitas unidades de saúde brasileiras, além de ser ineficaz quando desacompanhado de orientação e conscientização do prescritor, como já evidenciaram alguns trabalhos sobre o tema. 
Por outro lado, a simples obediência ao que preconizam as legislações existentes viabilizaria significantes melhoras no processo de prescrição e consequente dispensação. Porém, a redação de novas leis sobre o tema também suscita pouco ou nenhum efeito quando não há associação com campanhas educativas e de sensibilização dos profissionais prescritores.

Assim, sugere-se como bastante válida a oferta de palestras e de materiais informativos de fácil acesso, como folders, cartazes e cartilhas, tanto aos profissionais como também à população, para conscientizá-la quanto ao seu direito de receber uma prescrição médica adequada, que é amparado por legislações específicas.

\section{REFERÊNCIAS}

1. Odukoya OK, Chui MA. E-prescribing: A focused review and new approach to addressing safety in pharmacies and primary care. Res Soc Adm Pharm. 2013. 9: 996-1003.

2. Coustasse A, Shaffer J, Conley D, Coliflower J, Deslich S, Sikula A. Computer Physician Order Entry (CPOE): benefits and concerns - a status report. J Infor Tech Res. 2013. 6(3): 16-31.

3. Gilligan AM, Miller K, Mohney A, Montenegro C, Schwarz J, Warholak TL Analysis of pharmacists' interventions on electronic versus traditional prescriptions in 2 community pharmacies. Res Soc Adm Pharm. 2012. 8: 523-32.

4. Barreira PF, Sena OS, Brito AMG, Rios MC, Silva S. Prescrições medicamentosas: luz ou sombra para o usuário e farmacêutico. Rev Bras Farm. 2011. 92(4): 340-5.

5. Brasil. Lei no 5991 de 17 de Dezembro de 1973. Dispõe sobre o controle sanitário do comércio de drogas, medicamentos, insumos farmacêuticos e correlatos e dá outras providências. Diário Oficial da União. 19 de dezembro de 1973.

6. Brasil. Agência Nacional de Vigilância Sanitária. Resolução da Diretoria Colegiada n. 44 de 17 de agosto de 2009. Dispõe sobre Boas Práticas Farmacêuticas para o controle sanitário do funcionamento, da dispensação e da comercialização de produtos e da prestação de serviços farmacêuticos em farmácias e drogarias e dá outras providências. Diário Oficial da União. 18 de agosto de 2009.

7. Aguiar G, Silva Junior LA, Ferreira, MAM. Ilegibilidade e ausência de informações nas prescrições médicas: fatores de risco relacionados a erros de medicação. Rev Bras Promoç. Saúde. 2006. 19(2): 84-91.

8. Conselho Federal de Medicina. Resolução no 1897 de 6 de maio de 2009. Código de processo ético-profissional. Diário Oficial da União. 06 de maio de 2009.
A impressão de formulários de prescrição com os campos predeterminados para as informações cruciais, em lugar de uma folha apenas com o timbre da unidade, como ocorre na maioria dos locais, também poderia ser uma estratégia interessante, e mais factível à maioria das unidades de saúde brasileiras, que a implantação de sistemas de prescrição eletrônica.

\section{AGRADECIMENTOS}

Agradecemos à farmacêutica Herbenya da Silva Peixoto, responsável pelo serviço de Farmácia onde foi realizado o estudo, por sua colaboração no desenvolvimento da pesquisa.

9. São Paulo (Estado). Lei $n^{\circ}$. 10.241 de 17 de março de 1999. Dispõe sobre os direitos dos usuários dos serviços e das ações de saúde no Estado e dá outras providências. Diário Oficial do Estado de São Paulo. 19 de março de1999.

10. Paraná. Lei $n^{\circ} .13 .556$ de 14 de maio de 2002. Dispõe sobre obrigatoriedade de expedição de receitas médicas e odontológicas digitadas em computador, datilografadas ou escritas manualmente em letra de imprensa. Diário Oficial do Estado do Paraná. 15 de maio de 2002.

11. Amazonas. Lei Ordinária ${ }^{\circ} 3.199$ de 04 de dezembro de 2007. Dispõe sobre a obrigatoriedade de expedição de receitas médicas e odontológicas digitadas em computador ou escritas manualmente em letra de imprensa, forma ou caixa alta. Diário Oficial do Estado do Amazonas. 06 de dezembro de 2007.

12. Paraíba. Lei no .9373 de 03 de junho de 2011. Dispõe sobre a obrigatoriedade de expedição de receitas médicas e odontológicas digitadas em computador, datilografadas ou escritas manualmente em letra de forma ou caixa alta. Diário Oficial do Estado da Paraíba. 05 de junho de 2011.

13. Santos GEO. Cálculo amostral: calculadora on-line [Acessado 2012 jan 12]. Disponível em: http://www.glaubersantos.com/calculoamostral.html.

14. Brasil. Agência Nacional de Vigilância Sanitária. Portaria ${ }^{\circ} 344$ de 12 de Maio de 1998. Aprova o Regulamento Técnico sobre substâncias e medicamentos sujeitos a controle especial. Diário Oficial da União. 19 de Maio de 1998.

15. Moretto LD, Mastelaro R (Org.). Manual das Denominações Comuns Brasileiras. São Paulo: SINDUSFARMA. 2013.

16. Lins BG, Cazzamalli F, Zancanaro V. Análise de erros nas prescrições médicas de uma unidade básica de saúde de um município do meio oeste catarinense. Rev Interdiscip Estudo Saúde. 2012. 1(2): 62-77. 
17. Lyra Júnior DP, Prado MCTA, Abriata JP, Pelá IR. Recetas médicas como causantes de riesgo de problemas relacionados con medicamentos. Seguim Farmacoter. 2004. 2(2): 86-96.

18. Dantas JPN. Determinação do perfil dos erros em prescrições ambulatoriais atendidas em um hospital público de Icapuí- CE. [Dissertação]. Fortaleza: Escola de Saúde Pública do Estado do Ceará; 2008.

19. Guzzato P, Bueno D. Análise de prescrições medicamentosas dispensadas na farmácia de uma unidade básica de saúde de Porto Alegre-RS. Rev HCPA. 2007. 27(3): 20-6.

20. Frick GGG, Bonotto LF, Bernardi CLB, Pletsch MU. Principais problemas encontrados nas prescrições em município da região noroeste do Estado do Rio Grande do Sul. Contexto \& Saúde. 2010. 10(19): 108-11.

21. Brasil. Ministério da Saúde. Uso Racional de Medicamentos: temas selecionados. Brasília: Ministério da Saúde. 2012.

22. Samaranayake NR, Cheung BMY. Medication safety in hospitals: avoiding medication errors in the medication use process. Adv Pharmacoepidem Drug Safety. 2013. 2(3): $1-7$

23. Sebastião ECO. Avaliação do cumprimento das exigências legais em ordens médicas em serviço de farmácia hospitalar de Ouro Preto e implicações na qualidade assistencial ao usuário. Rev Bras Farm. 2002. 23(1): 71-85.

24. Néri EDR. Determinação do perfil dos erros de prescrição de medicamentos em um hospital universitário [Dissertação]. Fortaleza: Universidade Federal do Ceará. 2004.

25. Ivama AM, Noblat L, Castro MS, Oliveira NVBV, Jaramillo NM, Rech N. Consenso Brasileiro de Atenção Farmacêutica - Proposta. Brasília: Organização Pan-Americana da Saúde. 2002.

26. Rosa MB, Perini E, Anacleto TA, Neiva HM, Bogutchi T. Erros na prescrição hospitalar de medicamentos potencialmente perigosos. Rev Saúde Públ. 2008. 43(3): 490-8.

27. Araújo PTB, Uchoa SAC. Avaliação da qualidade de prescrição de medicamentos de um hospital de ensino. Rev Cien Saude Colet. 2011. 16 (supl 1): 1107-1114.

28. American Society of Health-System Pharmacists. ASHP guidelines on preventing medications errors in hospitals. In:

Best practice for hospital and health system pharmacy 2013-2014: Positions and guidance documents of ASHP. Bathesda: American Society of Health-System Pharmacists. 2013. 214-22.
29. Percly JS, Almeida MS, Bolzan MH, Pimentel KRFV, Luca M. Análise qualitativa de receitas ambulatoriais em dado período em um hospital da rede pública: possíveis fatores que podem influenciar o uso correto de medicamentos. Rev Bras Farm. 2006. 87(3): 89-91.

30. Lisby M, Nielsen LP, Mainz J. Errors in medication process: frequency, type, and potential. Int J Qual Health C. 2005. 17(1): 15-22.

31. Gimenes FRE, Mota MLS, Teixeira TCA, Silva AEBC, Optiz SP, Cassiani. Segurança de pacientes na terapêutica medicamentosa e a influência da prescrição médica nos erros de dose. Rev Latino-Am Enferm. 2010. 18(6): 1-7.

32. Ather A, Neelkantreddy P, Anand G, Manjunath G, Vishwanath J, Riyaz M. A study on determination of prescription writing errors in outpatient department of medicine in a teaching hospital. Indian J Pharm Pract. 2013. 6(2): $21-4$.

33. Avery AJ et al. The prevalence and nature of prescribing and monitoring errors in English general practice: a retrospective case note review. Br J Gen Pract. 2013. e543-53.

34. Sánchez AMM. Medications errors in a Spanish community pharmacy: nature, frequency and potential causes. Int J Clin Pharm. 2013. 35: 185-9.

35. Nanji KC, Rothschild JM, Salzberg C, Keohane CA, Zigmont K, Devita J, Gandhi TK, Dalal AK, Bates DW, Poon EG. Errors associated with outpatient computerized prescribing systems. J Am Med Inform Assoc. 2011. 18: 767-73.

36. Ekedahal A. Problem prescriptions in Sweden necessitating contact with the prescriber before dispensing. Res Social Adm Pharm. 2010. 6(3): 174-84.

37. Abramovicius AC. Estudos de erros de prescrição relacionados aos medicamentos utilizados no Hospital das Clínicas da Faculdade de Medicina de Ribeirão Preto [Dissertação]. Ribeirão Preto: Universidade de São Paulo; 2007.

38. Villamañán E, Larrubia Y, Ruano M, Vélez M, Armada E, Herrero A, Álvarez-Sala, R. Potential medication erros associated with computer prescriber order entry. Int J Clin Pharm. 2013. 35: 577-83. 